\title{
GESNERUS
}

Vierteljahrsschrift für Geschichte der Medizin und der Naturwissenschaften

Revue trimestrielle d'histoire de la médecine

Jahrgang/Vol. $22 \quad 1965$ Heft/Fasc. 3/4

\section{Conrad Geßners Persönlichkeit}

\section{März 1516 bis 13.Dezember 1565 \\ Von Charles Salzmann}

\begin{abstract}
Ahnherr der Familie Geßner war der Goldschmied Hans Gessner ${ }^{1}$ aus Nürnberg, der, vor 1500 (1481) in Solothurn eingewandert, das Bürgerrecht erwarb. Sein Sohn Andreas ${ }^{2}$ kam 1504 als Gewürzkrämer nach Zürich, ihm folgte 1511 der Bruder Urs, der Kürschner, nach. Urs Gessner heiratete in Zürich aus der Gemeinde Kappel Agathe FricK ${ }^{3}$, aus deren Ehe mehrere Kinder entsprossen, von denen Hans, Conrad und Elisabeth bekannt sind.

Am 11.Oktober 1531 in der Schlacht bei Kappel starben Huldreici Zwingli mit 25 geistlichen Herren, 26 Ratsherren Zürichs und an die 400 Bürger.

Urs Geßner fiel in den blutigen Treffen an Lorzenübergängen bei Baar oder auf dem Gubel (nördlich von Unter-Ägeri) am 24./25. Oktober, die den Zweiten Kappelerkrieg entschieden. Bei der hohen Kriegsentschädigung an die katholischen Orte litten viele Zürcher Familien Leid und bittere Not.

Die Mutter Geßners brachte den Conradli schon vor der Schulzeit (vor 1528) zu ihrem Bruder, dem Chorherrn Johannes Frick, an «Tisch und

1 Historisch-Biographisches Lexikon der Schweiz, Band 3, S. 498-500 (Geßner).

2 Andreas Gessner, 1484-1568, erlitt 14. Wunden bei Kappel. - C.D̈̈niker, Geschichte der Schweiz, Band 2, Zürich 1892, S. 533-537 (Kappel); TH. MüLler-Wolfer, Das Jahrhundert der Glaubenstrennung, Schweizer Kriegsgeschichte, Heft 5, S. 29-52. - Im Testament des großen Konrad Gessner, vom 18 ten Herbstmonat 1564, ist zu lesen: «Ursen Geßners, Kürßners u. Burgers zu Zürich, welcher in meiner Herren Nöthen auf dem Berg bey Zug oder Baar geblieben ist im Jahr 1531 », Patriotisches Archiv für die Schweiz, Erster Theil, Basel 1789, S. 95. - Ebenso bei J.Hanhart, Winterthur 1824, S. 285.

3 Agathe Frick, in J. Hanhart, Conrad Geßner, Winterthur 1824, S. 4. - Barbara Frick, in Bibliotheca universalis, 1545.
\end{abstract}


Bett», wo er liebevolle Aufnahme fand; hernach sorgte drei Jahre Јон. $\mathrm{JaK}_{\text {. AmmanN }}{ }^{4}$ für ihn.

Die Ernährung muß damals eine kärgliche gewesen sein, bei wenig Gemüse und Früchten; dafür gab es Hirse- und Hafermus und, wenn es gut wollte, in der Woche ein- bis zweimal Fleisch, oft auch keines; etwas Milch und Käse, Eier und Fisch waren Zehntengaben der Chorherren zu Festtagen und daher selten. Das Brot, meist Roggen- und hartes Gerstenbrot, wurde den Hausgenossen zugemessen als Hungerstiller. Im ganzen war diese Ernährung für Jugendliche ungenügend. Im Winter 1531/1532 erkrankte Conrad Gessner, wie er dies in der eigenen biographischen Skizze in der Bibliotheca universalis bekundet: (S. 180 « ego aliquandiu aqua inter cutem afflictus»-) «Ich selbst lag einige Zeit an einer Wassersucht krank» (vermutlich Hungerödem).

Noch anfangs Dezember 1565 ist in einem Brief ${ }^{5}$ an den Freund AdolpH Occo $^{6}$ in Augsburg zu lesen: "Ich war von Jugend auf blaß, schmächtig und von schwächlicher Gesundheit.»

An der Fraumünsterschule wurde ihm in Oswald Myкonius ${ }^{7}$ ein zweiter Vater, dem er sich von nun an voll vertraute, der seine reichen Fähigkeiten richtig erkannte.

Dieser gütige Lehrer empfahl ihn schon im Frühjahr 1532 an WoLfGANG Fabricius CAPIto $^{8}$ zu Straßburg; er nannte C.Geßner «einen geistvollen

4 J.J.Ammann, 1500-1573, Professor für Latein, Griechisch, Logik, Rhetorik, 1524, Chorherr in Zürich, in Historisch-Biographisches Lexikon der Schweiz, Band 1, S. 344. In Јон. Н.НоттіNGERS Kirchengeschichte wird ein frivoler Scherz überliefert, der das

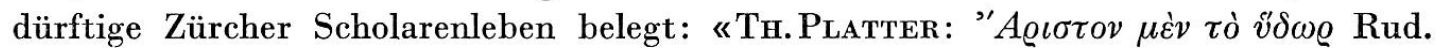
Collinus: Age dum bibamus aquam cum vinum desit. Pauca sufficiunt illi, qui pauca desiderat.» - Heinrich Boos, Thomas und Felix Platter, Leipzig 1878, S. 51: «einest als wier znacht aßen, by dem wasser krûg, sprach er: «Platere, wie facht Pindarus an»?

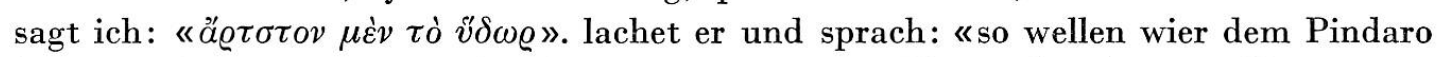
folgen und so wier nit win hand, wasser trinken». - Ebenso bei Alfred Hartmann, Thomas Platter, Lebensbeschreibung, Basel 1944, S. 77.

5 Hanhart, a. a. O., S. 275-277.

6 Adolph Occo III., 1524-1606, Arzt in Augsburg, promovierte 1549 zu Ferrara, 1563 Archiater von Augsburg, Numismatiker, 1572 von Kaiser Maximilian II. geadelt. In Conradi Gesneri Epistolarum medicinalium, Liber secundus, T. 1577, S. 29, 36, 36a, $35,33 \mathrm{a}, 37,39,40 \mathrm{a}, 41$.

7 Oswald Mykonius, genannt GeIsshüsler: 1488-1552, in Historisch-Biographisches Lexikon der Schweiz, Band 3, S. 425; Hanhart a. a. O., S. 6, 14-15.

8 Wolfgang Fabricius Capito, 1478-1541, in Historisch-Biographisches Lexikon der Schweiz, Band 2, S. 488; Hanhart, a.a.O., S. 20-42. - Otto Erich Strasser, Capitos 
Jüngling, ein so vortrefflicher Kopf, der nicht zugrunde gehen müsse, ich habe mehr seine Fortschritte bewundert, seine großen Anlagen, er ist gut, er lernt mit Lust ... Du wirst ihn sehr lenksam finden ... er ist von langer und hoher Gestalt, er ist erst sechzehn Jahre alt.»

Im Juni 1532 wanderte Geßner nach Basel und Straßburg zu W.F.Capito. Er fühlte sich freier in freudiger Hingabe zum Studium des Hebräischen, aber als «Famulus» fand er wenig Zeit bei Hausgeschäften zum Lesen von Lateinern und Griechen. Trotzdem entstand in dieser Stimmung eine kleine Sammlung von griechischen Elegien auf Huldreich Zwingli.

Seit den ersten Schuljahren besaß C. Geßner eine leichte Auffassungsgabe, eng verknüpft mit der Begeisterung für alles Wissenswerte, was sich ihm bot, verbunden mit jener großen Aufgeschlossenheit, die ihn frühzeitig auszeichnete, in einem zeitlich kaum beschränkten Erinnerungsvermögen von bester Treue über Jahrzehnte.

In seiner Lernfähigkeit lag ihm die altgriechische Sprache an erster Stelle, von der er sagte, er beherrsche sie wie sein Zürichdeutsch.

Heinrich Bullinger ${ }^{9}$ ermöglichte ihm und JoH. Fries einen zweijährigen Aufenthalt in Frankreich.

Die philologischen Studien vertiefte C.Geßner in Bourges en Bresse (einer damals nicht unbedeutenden Universität) unter Anleitung des anerkannten Gräzisten WilHelm VolmaR ${ }^{10}$ aus Bern. Schon am 14.April 1533 schrieb er an O.Mykonius: "Wir sind zwar gesund und wohlbehalten in Bourges angekommen, aber wahrlich, hier lacht uns nicht alles an. Alle Wissenschaften sind hier vernachlässigt, nur die Rechtsgelehrsamkeit nicht, denn diese wird des Alciatus wegen in großen Ehren gehalten.» Im gleichen Ton klingt sein Brief an H.Bullinger: «Könnten wir die Musen von Bourges ebensosehr rühmen wie unsere Gesundheit, so stünde es trefflich um uns. Doch nie haben die Götter den Menschen alles zusammen gegeben. Hier blüht das bürgerliche Recht.»

An der Universität Bourges dozierte von 1529 bis 1533 der Mailänder Rechtsgelehrte Andrea Alciatus ${ }^{11}$ glänzend das römische Recht «en beau langage latin des lettres humaines avec le droit».

Beziehungen zu Bern, Diss. Bern, Gräfenhainichen 1927, wertvolle Quellen und Literaturangaben.

9 H.Bullinger, 1497-1575, Nachfolger Zwinglis, Antistes, Historisch-Biographisches Lexikon der Schweiz, Band 2, S. 424.

10 Wilhelm Volmar (alias Melchior Rot, Rubellus), früher Bürger von Rottweil, 1497-1561, Historisch-Biographisches Lexikon der Schweiz, Band 7, S. 294.

11 Andreas Alciatus, 1492-1550 aus Alzate bei Como, bedeutender Rechtsgelehrter, mit 
Geßner fand Unterkunft im Hause Volmars, dazu das «glückliche Los», dessen Söhne in Latein zu unterrichten, somit war er finanziellen Sorgen enthoben. Eine reichliche Zeit verblieb ihm zur Muse, zum Lesen griechischer und lateinischer Historiker, Dichter, Philosophen, ganz besonders griechischer Ärzte, wie er sie im Bücherkasten des MeLchior Volmar aussuchte. Unter den Schülern Volmars traf er TheOdore DE BEZA ${ }^{12}$ und den Grafen Froben Christoph von Zimmern ${ }^{13}$, dessen Tante Catharina von Zimmern (einst Äbtissin) ${ }^{14}$ er von seinen Knabenjahren her, als er die Fraumünsterschule besuchte, kannte. Nach wenigen Monaten verließ ihn der ihm brüderlich zugetane Johannes Fries, da dieser keine Anstellung fand, um die «libera studia» zu bezahlen, und reiste nach Paris.

Im Frühling 1534, folgte ihm Geßner nach, wo ihn vor allem das große Talent des Hellenisten Guillaume Budé ${ }^{15}$, des «fondateur du collège de France», anzog, neben den Brüdern Estienne, den Buchdruckern. Er muß auch den vielseitigen Charles Estienne ${ }^{16}$, den Anatomen, Botaniker und

seiner Vorlesung: «De verborum significatione» an der Universität Avignon 1520/21 erlangte er Weltruf; zu seinen Schülern zählte neben C. Geßner auch Bonif. Amerbach. Seine schon 1522 edierten: Emblemata mögen ganz wesentlich C. Geßner zu seinen Tierbüchern angeregt haben. - H. De Giacomi, Andreas Alciatus, Basel 1934.

12 ThÉodore de BÈze von Vézelay, 1519-1605, Historisch-Biographisches Lexikon, Band 2, S. 220-221. - TH. DE BÈze erinnerte sich an C.Geßner: «cuius ut amicissimi et sub Melchiore Volmario communi praeceptore Biturigibus suavissimi condiscipuli obitum

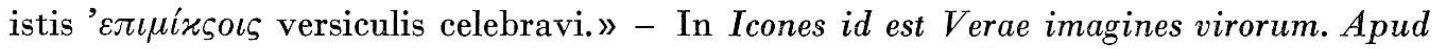
Joannem Laonium 1580.

13 Graf Froben Christoph von Zimmern, 1519-1566, Diss. Zürich 1959, von Beat RudolF JENNY.

14 Catharina von Zimmern, 1478-1545 ?, Historisch-Biographisches Lexikon der Schweiz, Band 7, S. 664, Äbtissin des Fraumünsters von 1496 bis 1524, verheiratet mit Ritter Eberhard v. Reischach, gefallen bei Kappel. Gerold Meyer v. Knonau, Die letzte Äbtissin des Fraumünsterstiftes in Zürich, Zürich 1876.

15 Guillaume Budé (Budaeus), 1468-1540. Eugène de Budé, Vie de Guillaume Budé (1467-1540), Paris 1884.

16 Charles Etienne, 1504-1564, studierte bei Jacques Dubois in Paris 1533-1536, Lauréat à Paris 1542, bekannt durch De la Dissection des Parties du Corps humain, 1543 und 1545. - Mit Henri Etienne II., 1531-1598, gemeinsam veröffentlichte C. Geßner die Editio princeps des Lexikon des Erotianus (1.Jh.n. Chr.) eine Zusammenfassung aus Hippokrates, Galen, Aretaeus, Oribasius und anderen alten Autoren mit Annotationen und Emendationen. Dictionarium medicum, vel expositiones vocum medicinalium. Henry Etienne, Genevae $1564,8^{\circ}, 318 \mathrm{~S}$. Dies ist eines der Werke, die zum Drucke gelangten mit der finanziellen Unterstützung des Ulrich Fugger (1526-1584). Vide OsLer 7028. 
Buchdrucker, kennengelernt haben. Noch nach vielen Jahren stand er in Beziehung mit Henri und Robert Estienne.

Während dem beinahe einjährigen Pariser Aufenthalt muß Geßner den Wert des Griechischen deutlich erkannt haben, was ihn in den folgenden Jahren zu einem der besten Kenner der antiken Literatur erhob und ihn wahrscheinlich gemeinsam mit dem Drucker Christoph Froschauer zur Bibliotheca universalis anregte. Er sah sich an den Anfängen der Lexikographie gemeinsam mit G.Budé, dem Verfasser der Annotations sur les Pandectes (1508) und Le traité des Asse (1514) ${ }^{17}$.

Bourges vermittelte ihm den gewünschten Einblick in die griechische Literatur, indes das Pariser Jahr ihm eine Fülle von neuen Eindrücken schenkte. Die öffentlichen Vorlesungen in Anatomie und galenischer Medizin, die Jaсов us Sylvius dozierte, dann die vielen Bücher, die in der engen Rue Saint-Jacques im Quartier latin feil waren, was muß er hier alles freudig gesucht haben!

In der sommerlichen Glut verließ er Paris, auf einem Seineschiff wohl, erreichte Rouen mit dem Port maritime und Dieppe, dem weiten Sandstrand, den hohen Falaises.

Noch nach dreißig Jahren erinnerte sich Geßner an den «Chruselbeerenstrauch», den "Ceanothus spina Theophrato memorata: Huius plantam vidi Diepae in Gallia, quae in arbusculum evaserat, et fructum Cerasi magnitudine serebat, haec cum ramosa atque frondosa esset, circumduxerat illi rete, atque ita aviculas in ea pulchro spectaculo nutriebant. ${ }^{18}$

Angesichts des Atlantiks und des schimmernden Horizonts muß ihn gewiß eine Seereise nach England und Schottland gelockt haben, denn in Horti Germaniae (S. 251 b) ist zu lesen: «Carduus hortensis (der Artischau, Welschdistel): sed postquam Luteciae et in Scotia carduos omnes fermè sine spinis esse cernebam» - somit steht die Frage einer Seereise nach Schottland offen.

Die Studienzeit in Paris nützte er bei dem Andrang vieler Möglichkeiten recht fruchtbar aus, die ihn mächtig anregten, alles an griechischen Werken zu sammeln; daher wuchsen die Auslagen durch den Ankauf von Büchern sehr beträchtlich.

17 Alfred Gudeman, Grundriß der Geschichte der klassischen Philologie, 2. Auflage, Leipzig/Berlin 1909, S. 188-189 (Budé).

18 Horti Germaniae, authore Conrado Gesnero, Argentorati 1561, S. 252. 
In dieser pekuniären Not half ihm der Berner JoH. Steiger ${ }^{19}$ freundeidgenössisch aus.

Wie es um ihre Geldnot, der «rerum summa necessitas», bestellt war, be-

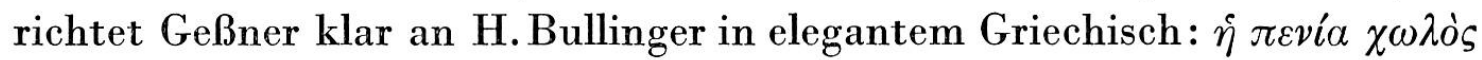
$\pi \varrho o ̀ \varsigma ~ \tau o ̀ ~ \varphi ı \lambda о \sigma o ф \varepsilon \tilde{v ~(d i e ~ N o t ~ l a ̈ h m t ~ z u d e m ~ d a s ~ P h i l o s o p h i e r e n) . ~}$

Ende November 1534 erlebte er jene blutigen Tage der zweigeteilten Christenheit ${ }^{20}$ in ihrer ganzen Tragik, um die Erneuerung des Glaubens, das Suchen nach religiöser Größe, die ihn in späteren Jahren noch bewegte. Er verließ Paris, durcheilte die Champagne und Lothringen, im Strom der Flüchtlinge erreichte er Straßburg, wo ihn Martin Bucer ${ }^{21}$ aufnahm. In einem Brief vom 27. Dezember $1534^{22}$ an H. Bullinger schildert er die blutigen Vorgänge in Paris, die Verfolgungen und Martern der Freunde der Reformation, aber auch seine Ausgaben und die großen Schulden ${ }^{23}$.

Heimfremd in Zürich angekommen, heiratete Geßner, kaum neunzehn Jahre alt, anfangs 1535 ein armes schönes Mädchen ${ }^{24}$, so arm wie er selbst, aber voller Lebensmut, «deren gute Eigenschaften und Liebe zu mir allein mich für sie eingenommen haben»; mehr findet sich nicht verzeichnet.

Diese frühe Vermählung, dazu die ansehnlichen Schulden wurden ihm von der kirchlichen Obrigkeit, welche das Stipendium ${ }^{25}$ verliehen hatte,

19 Joh. Steiger, 1519-1581, von Bern, Historisch-Biographisches Lexikon der Schweiz, Band 6, S. 520.

20 Placard-Verfolgung von Oktober bis Dezember 1534 in Paris.

${ }^{21}$ Martin Bucer, 1491-1551, Dominikaner, Reformator, Theologe, Historisch-Biographisches Lexikon der Schweiz, Band 2, S. 383-384.

${ }^{22}$ G.BudÉ erwähnt Geßner im Brief an H.Bullinger vom 27. Dezember 1534: «Er ist ganz der unserige », das berühmte: «Noster est totus»; G. BudÉ starb aber als resignierter Katholik, wie er es in seinem Testament angeordnet hatte: «je veux estre porté en terre de nuict et sans semonce, à une torche ou à deux seulement, et ne veux estre proclamé ni à l'église, ni par la ville, ni alors que je seray inhumé, ni le lendemain; car je n'approuvay jamais la coustume des ceremonies lugubres et pompes funèbres que l'on faict mesmement pour tels personnages que moi.»- Dies entsprach ganz der Auffassung des Aurelius Augustinus in De civitate Dei.

${ }^{23}$ Geldnot in Paris: Epistola C. Gesneri ad Bullingerum, September 1534, in Epistolarum medicinalium, Liber quartus, in HANHART, a.a. O., S. 346-347.

${ }^{24}$ Hanhart, a.a. O., S. 42-56, Epistola ad H.Nüscheler, 24. März 1535: formosa puella. In Bibliotheca universalis, S. 180: "paulo post intempestivius uxore ducta -» Betreffend der Frau Geßners vide Eugène Olivier, Les années lausannoises (1537-1540) de Conrad Gessner, in Revue suisse d'histoire 1 (1951) 374-375 : Barbara Singysen, vermutlich aus Mellingen; im Testament: Barbara Pantli, noch 1579, im Neujahrsblatt 1819.

${ }^{25}$ Das Stipendium für Geßner betrug für 1533/34 6 Mütt Kernen und $28 \mathrm{fl}$. 
recht übel genommen. Mit Bitterkeit notierte er hiezu: «Und ich wurde in einen Winkel der öffentlichen Schule geworfen, wo ich eine Zeitlang um ein kleines Gehalt, das mir nichts besseres als Hungers sterben hoffen ließ» (Bibliotheca universalis, S. 180).

Der Praeceptor litterarum zeigte keine Lust, in der Schulstube auszuhalten, ihn begeisterte das Studium der Medizin; nachts las er griechische Ärzte, tags neben den Schulstunden verdiente er als Korrektor in den Buchdruckereien sein hartes Brot.

In dieser Not schrieb er an den väterlichen Freund Mykonius ${ }^{26}$ in Basel: «Ich habe eine große Begierde, die Kräfte der einfachen Heilmittel sicher kennenzulernen, um meinen Kranken und mir besser raten zu können. Erst dann kann ich auch in meinen Schriften nicht nur das von andern schon Gesagte wiederholen; auch werden eigene Beobachtungen besser und sicherer an unserem eigenen Körper gemacht als an Freunden.»

Der Segen des Apollinischen durchglühte ihn, bewahrte ihn vor Daseinsfurcht, jeder Angst vor Gefahr in seinen Experimenten, ganz im Sinn, wie er an Jacques Delechamps ${ }^{27}$ schrieb: «Ego sum via, veritas et vita, quis aliam praeter Christum viam ostendit? ${ }^{28}$

In der Bibliotheca universalis (S. 180a) bemerkt er: «Cum a puero ingenium meum in medicinae studium proclivo feretur.»

Nahezu zwei Jahre dauerte dieses Schulexil Geßners, trotz etlicher Briefe an Mykonius in Basel; er seufzt: «Kannst du noch etwas für mich tun, so suche mir doch wenigstens einen Beschützer zu verschaffen.Bullinger ist mir nicht geneigt; ach, wie oft sehne ich mich seufzend nach meinem Zwingli.» Oswald Mykonius' wiederholte Bitten, vereint mit denen J.J. Ammanss, bewegten H.Bullinger, ein angemessenes Stipendium für Geßner zum Medizinstudium in Basel zu erwirken.

Kaum in Basel (20.Dezember 1536) erhielt Geßner den ersten großen Auftrag von Henric Petri senior ${ }^{29}$, die Bearbeitung eines griechisch-lateinischen Lexikons, dessen Druck ihm keine Freude bereitete, da der

${ }^{26}$ Hanhart, a. a. O., S. 44-60, Epistolae ad Mykonium, April 1536, 12. Juni 1536, 3. Juli 1536, 30. Juni 1535.

27 Jacques Delechamps, né à Caen 1513, mort à Lyon 1588, Médecin, Botaniste. Larousse $d u X X^{e}$ siècle, Tome II, p. 653; Dictionnaire des sciences médicales, Biographie médicale, Tome III, p. 376-377.

${ }^{28}$ Conradi Gesneri Epistola: De Constantia in Fide, S. 137, in Museum Helveticum, Particula I, Tiguri 1746.

29 Henric Petri, 1508-1579, Dr. med., Buchdrucker und Verleger in Basel, HistorischBiographisches Lexikon, Band 5, S. 409-410. 
Drucker manche seiner griechischen Erklärungen als überflüssig wegließ: «Weil sie dieselben für meine Arbeit hielten, deswegen verwarfen, da doch dieselben aus dem Phavorinus, dem Hesychius, dem Suidas und anderen griechischen Kommentaren ausgezogen waren, obgleich der Herausgeber in seiner Vorrede dieses Vorzuges mit keinem Worte erwähnte, um denen nicht nachteilig zu werden, welche zu gleicher Zeit in Basel das große Lexikon des Phavorinus in Druck herausgaben. Doch weil jener Buchdrucker und die, welche so grausam, so ungerecht mit meinem Wörterbuche umgingen, gestorben sind, so sage ich weiter kein Wort hierüber» (Bibliotheca universalis, S. 180-180 a).

Auf Empfehlung von Mykonius beriefen ihn die Magistraten Berns als Griechischprofessor an die neugegründete Akademie nach Lausanne.Wenn ihn auch der Aufschub der Medizinstudien bedrängte, die Schulden drückten, selbst das Heimweh schmerzte, so freute er sich, der größten Sorgen enthoben; hier besaß er ein eigenes Haus, dazu ein recht ansehnliches Honorar (etwa 8000 Franken, im Wert von $1925^{30}$ ).

Die Lausanner Jahre (Oktober 1537 bis Oktober 1540) müssen für Geßner eine glückliche und fruchtbare Zeit gewesen sein. In den Morgenstunden erklärte er den Demosthenes, Isokrates, den Homer, Pindar, Sophokles, Euripides, in den Nachmittagsstunden die Ethik des AfistoteLES und die Politaia, die Dialoge des Plato. In den Ferien durchstreifte er herborisierend das Waadtland, die Höhen am Mont Jorat, entlang den Ufern des Léman. Im August 1539 reiste er mit seiner Gattin ins Wallis in das Leukerbad, er bestieg die Gemmi, suchte nach seltenen Pflanzen in den Bergen. Eine unbändige Wißbegierde beflügelte ihn zum Studium der Pflanzenkunde eines Dioscorides, Theophrastos, Plinius, Paulus AegiNeta, Aretaios in diesen Jahren, die er in den folgenden Jahren (1540 bis 1542 ) in kleineren Auszügen veröffentlichte ${ }^{31}$. Jedes dieser Werklein stattete er in liebenswürdiger Weise mit einem Dedikationsbrief an einen seiner Freunde, Gönner, Lehrer, Bekannten aus als Dankesadresse für eine Gunst, Hilfe oder Zuneigung, die dem Leser heute noch eine beglückende Einsicht in das Wesen Geßners gewähren ${ }^{32}$.

Von Lausanne zog Geßner allein anfangs Oktober 1540 nach Montpellier, der damals berühmtesten Medizinschule, zur Fortsetzung der medizinischen

30 Eugène Olivier, Les années Lausannoises 1537-1540 de Conrad Gessner, Revue suisse d'histoire 1 (1951) 369-428, besonders S. 373.

31 Bibliotheca universalis, S. 180.

32 HANHART, a.a.O., S. 60-61, 67-78. 
Studien unter angesehenen Ärzten, vorab dem Guillaume Rondelet ${ }^{33}$, dem Freund Françors Rabelais ${ }^{34}$, einem gewandten Anatomen, Ichthyologen und Botaniker, also einem Mann ganz nach dem Herzen Geßners. Über den jüngeren Dozenten Antoine de Saporta fehlen Angaben von Geßners Hand.

Mit einem spanischen Arzte, Peter Jakobus, einem guten Pflanzenkenner, dem er das Compendium ex Actuarii Zachariae libris de differentiis urinarum, indiciis, causis et praevidentiis $\left(8^{\circ}\right.$, Tiguri 1541) widmete, durchwanderte er die Provence, die Küsten des Mittelmeeres. Von diesen Streifzügen ans Meer mag jene Koralle stammen, wie sie sich abgebildet im Fossilienbuch findet.

Noch vor Anbruch des Winters nahm er enttäuscht Abschied von Montpellier: «Allein ich mußte den so sehr gewünschten Anlaß zu näherem Umgang mit den berühmtesten Ärzten, den ich ihren Vorlesungen vorzug, missen. Nachdem ich in der Anatomie und der Kräuterkunde einige Fortschritte getan hatte, gab ich den dortigen Aufenthalt auf.» ${ }^{35} \mathrm{~W}$ as er suchte,

33 Guillaume Rondelet, 1507-1566, Schüler Gunther D'Andernachs, bedeutender Ichthyologe; er schrieb: De piscibus marinis, Lugduni 1554-1555, mit 422 Holzschnitten.Antorne Saponta, Prof. med. in Montpellier 1540, Dekan 1551, Kanzler 1566. Antoine Saporta war 1552 und die folgenden Jahre Lehrer von Felix Platter. - Siehe bei H. Boos, Thomas und Felix Platter, Leipzig 1878, S. 191, 200, 220 f., 227, 230, $237 \mathrm{f}$, 258, 267 f. - Achilles Burckhardt, Thomas Platters Briefe an seinen Sohn, Basel 1890, S. $46,47,48,52,58,60,74,75,87,88,91$.

${ }^{34}$ François Rabelais, 1483-1553, er nannte Rondelet in seinen Werken «Rondibilis».

${ }^{35} \mathrm{Zu}$ seinen Studien in Montpellier bemerkt Geßner in der Epistola vom 6. Oktober 1560 an J. Crato von Krafftheim in Epistolarum medicinalium, Liber primus, S. 6 b: De schola Monspeliana non possum ut vellem iudicare, in re medica nihil tam illio quam Anatomica et stirpium historiam uberius quam in aliis gymnasiis Monspellii addiscere Philosophiae et Graecarum literarum nulla illic ratio. Si videbitur hortaberis ut hac hyeme diligentior sit et quicquid Monspelli disci potest, addiscat. - JoH. Сrato von KrafftHEIM, 1519-1586 aus Breslau stammend, studierte in Wittenberg, Schüler und Freund M.Luthers und Melanchthons, Studienfreund des bedeutenden Pharmakologen Valerius Cordus, Student und Dr. medicinae von Padua. Praktizierte 1550 in Augsburg, wo er an den Hofstaat Kaiser Karls V. kam, in der Folge Leibarzt Ferdinands I., Maximilians II., Rudolf II. als «intimum nostrum medicum et consiliarium» hoch geschätzt als Arzt, Berater und Höfling von überragender humanistischer Bildung. - Ihm verdankte Conrad Geßner sein Bemühen und Empfehlen um den Wappenbrief. Rühmlich sind die überlieferten Worte Ferdinands I. an J.Crato: Credas, Gesnerus tota probitas. Seine Consiliorum et episolarum medicinalium libri VII. 1592-1611 eine der reichhaltigsten Briefsammlungen. - In J.SimLer, Vita C.Gesneri, Tiguri 1566, S. 6 a «qui se domi hospitem susciperet, existimabat enim, quidem vere, ex domestica insignis 
fand er nicht: den persönlichen Kontakt mit den Ärzten, den Lehrern eine lachende Wahrheit (Simler, 6a). In Lyon verbrachte Geßner einige Tage als Gast bei dem Buchdrucker Joh. Frellonius; in Dankbarkeit überließ er ihm zum Druck drei kleinere Traktate, den Apparatus et delectus simplicium medicamentorum, de compositione medicamentorum secundum genera ex Paulo Aegineta, de compositione medicamentorum secundum genera universalis methodus ex Galeno, die er bereits in Lausanne ausgearbeitet hatte (erschien erst Lugduni 1542). Ein Dedikationsbrief an Christoph Clauser in Zürich ist datiert von Lyon, den 13. Januar 1541; daher wird ersichtlich, daß Geßner erst gegen Ende Januar 1541 Basel erreichte, mit Eifer die Vorlesungen besuchte, wo ihn im Februar 1541 Dekan Albanus Torinus ${ }^{36}$ zum Doktor promovierte.

Es ist erstaunlich, wie Geßner ohne geregelten Studiengang, nach wenigen Vorlesungen, vor allem im Selbststudium, sich in kaum drei Jahren aus den Schriften griechischer Ärzte und Naturforscher ein solch umfassendes ärztliches Wissen erwarb. Seit seinen Reisen nach Bourges, Paris, durch die Provence, Italien, Venedig, Padua erwachten in ihm aus der Schau der literarischen Reste der Antike jene humanistischen Kräfte; die erlebten Landschaften erhellten das Verständnis für die Pflanzen- und Tierwelt. Aus all diesen Eindrücken und Erlebnissen heraus formte sich seine Persönlichkeit, den Worten Marcus Tullius Cicero folgend: «Virtus enim laus omnis in actione consistit!» (de officiis I. 19).

Am Anfang seines gewaltigen Arbeitsanlaufes steht die Bibliotheca universalis $^{37}$, die Frucht jahrelanger, fleißigster Arbeit, von einer erstaunlichen Genauigkeit, ein Behältnis geistigen Schaffens, ein Grundwerkzeug des Wissens. Für das Biographische, für das Persönliche, die Eigenart des Menschen besaß er jenen frühreifen Sinn, neben dem Maß des Wertes ihrer Werke, all dies galt ihm wissenswert. Von all dem zeugen kurze persönliche Bemerkungen in der Bibliotheca universalis. Seine edle Gesinnung und Aufrichtigkeit lesen wir in der Zueignung der Bibliotheca universalis an CHRIStOPh Froschauer ${ }^{38}$ :

alicuius medici consuetudine, plus sibi doctrinae accessurum esse, quam ex eiusdem vel quotidianis publicis praelectionibus. - Crato von Krafftheim in Studien zur Geschichte der Medizin, von Viktor Fossel, Stuttgart 1909, S. 24-45.

36 Albanus Torinus, 1489-1550, Professor der Medizin in Basel.

37 Bibliotheca universalis, Tiguri 1545.

38 Christoph Froschauer, etwa 1490-1564, Historisch-Biographisches Lexikon der Schweiz, Band 3, S. 347-348. 
«Den ersten Platz aber unter den berühmten Typographen unserer Zeit habe ich dir, mein Froschauer, angewiesen, denn du warst es vor allen, der mich antrieb, die beiden Teile dieser Bibliotheca zeitig auszuarbeiten, und du hast sie, wie auch die meisten meiner übrigen Arbeiten, mit viel Kosten und Mühe durch den Druck veröffentlicht. - Du, bester Froschauer, nimm dieses erste Buch freundlich an und fahre fort, die Wissenschaften, besonders die Grammatik und die Philologie, wie sie es verlangen, mit Eifer zu fördern. ${ }^{39}$

Geßners Lebenslauf läßt sich nahezu anhand seiner Briefe und an denen seiner Freunde, verbunden mit der Durchsicht seines großen Schrifttums, ablesen.

Zeit seines Lebens suchte er nach Büchern, Codices, Manuskripten, Pergamenten in Paris, Venedig, in Augsburg, bat er Drucker um ihre Kataloge, arbeitete um der Bücher willen; manche müssen ihn besonders gefreut haben, nicht daß er im heutigen Sinn Bibliophiler gewesen wäre. Er durchforschte Bibliotheken nach griechischen Werken, um sie zu veröffentlichen. Der Freund Jон. Fries ${ }^{40}$ brachte ihm aus der Bibliothek des spanischen Granden und Diplomaten Karls des V. Diego Hurtado de Mendoza ${ }^{41} 1545$ aus Venedig die Melissa, eine Sammlung griechischer Kirchenväter, die er schon im folgenden Jahr im Druck lateinisch herausgab. Für die Gartenstudien fand er die Opera Joanni Pontani, De hortis Hesperidum ${ }^{42}$. Und Michael Schütz (Toxites) ${ }^{43}$ überbrachte ihm den Codex Palatinus, der die Bücher Marci Aurelin Antonini in semet ipsum enthielt aus der Bibliothek des Herzogs Otto-Heinrich von der Pfalz. Von Bonifacius

39 Paul Leemann-van Elck, Die Offizin Froschauer, Mitteilungen der Antiquarischen Gesellschaft in Zürich, Band XxxIII, Heft 2, Zürich 1940; Zürcher Drucker um die Mitte des 16. Jahrhunderts, Bibliothek der Schweizer Bibliophilen, Bern 1937.

40 ERnst Walder, Reise von Zürchern nach Venedig vor vier Jahrhunderten (Joh. Fries 1545), in Zürcher Taschenbuch auf das Jahr 1927, Zürich 1926, S. 159-184.

41 Diego Hurtado de Mendoza, geboren 1503 zu Granada, gestorben 1575 zu Madrid, im Dienste Karls des V. in Venedig; er besaß eine ansehnliche Bibliothek, die ArNold ArLenius verwaltete. - ED. Fueter, Geschichte der Neueren Historiographie, München/ Berlin 1911, S. 239-240 (D.H.de Mendoza). - Antonir Loci Melissa inscripti, inq. duos Tomos divisi numero sunt 175, Tiguri 1546 (Kleinformat).

42 Joanno Pontani, in Opera: De hortis Hesperidum libri duo. Vence in aedibus Aldi Manutii et Andreae soceri, 1533. - Joanno Pontano, 1426-1503, italienischer Humanist, Geßner zitiert ihn in Horti Germaniae, S. 254: de citrorum cultu Jovinianus Pontanus libros 2 carmine condidit.

43 Michael Toxites (alias Schütz), 1515-1581. - C. Schmidt, Michael Schütz, gen. Toxites, Straßburg 1888. 
Amerbach ${ }^{44}$ in Basel erbat er den Traktat Philobiblon des Richard De BuRY ${ }^{45}$; indessen mehr erhoffend, schrieb er von seinem Gönner ANton FUGGER ${ }^{46}$ : «et ne forte mireris, quid tanto viro mecum sit », dann folgt eine leise Bitte um eine Gunst: «Vale et nugis meis sive puerilibus, si ita videtur, studiis fave. - Des. Erasmi felicis memoriae librorum indicem a Gilberto Cognato accepi. Iterum atque iterum vale!»

In evangelischer Demut, erwachsen im Schatten der Münstertürme Zürichs, berührte ihn stets ein tiefes religiöses Empfinden, jede mystische Frömmlerei der Schwarmgeister war ihm fremd. Vom calvinistischen Geiste seit Lausanne beeindruckt, der ihn verinnerlichte, bis zu Aristoteles De Anima ${ }^{47}$ und den frühen griechischen theologischen Schriften, vor allem die von ihm übertragenen Epistolae Ignati Martyris ${ }^{48}$, folgte er dem

44 Bonifacius Amerbach, 1495-1562, Jurist, Professor in Basel, Historisch-Biographisches Lexikon der Schweiz, Band 1, S. 338. - Hanhart, a. a. O., S. 347-348 (Epistolarum medicinalium, Liber quartus).

45 Richard D'Aungerville, genannt De Bury, Bischof von Durham, in Northumberland, 1287-1345, Verfasser des Philobiblon. - Philobiblon - Traktat des Richard de Bury über die Liebe zu den Büchern, Leipzig 1911.

46 Anton Fugger, 1493-1560, Finanzmann und Kaufmann mit großen humanistischen Interessen.

47 C. Gesneri, De Anima, liber, Tiguri 1563.

48 C. Gesneri, Antonii loci «Melissa» inscripti numero sunt 175, Maximi vero 71, Abbae Maximi, philosophi, confessor et Martyris, Aphorismorum seu capitum de perfecta charitate et aliis virtutibus christianis ad Elpidium prespyterum Centuriae III. Theophili sexti Antiocheni episcopi de Deo et fide christianorum contra gentes institutionum libri tres ad Autolycum. Tatiani Assyrii Justini Martyris discipuli oratio contra Graecos. Tiguri, apud. Ch. Froschoverum 1546. kl. Fol. Jон. Fries brachte C. Geßner die «Melissa» von Venedig 1545 aus der Bibliothek des Diego Hurtado de Mendoza. - Veterum aliquot Theologorum Graecorum orthodoxorum libri Graeci - iidem latinitate donati e regione, Tiguri, apud Andream Gesnerum, 1552, ebenso noch 1559, 4. - Aeneae Gazei Platonici Theophrastus sive de animarum immortalitate. Ignatii Martyris et episcopi Antiocheni epistolae duodecim, Andrea Gesner, Tiguri 1559, $4^{\circ}$. - Einzelne dieser Tractate veröffentlichte C. Geßner aus einem Manuskript, das er in Augsburg 1545 nach Zürich brachte. Hanhart, a.a. O., S.166-168: John Ponetus, Bischof von Winton, bat H.Bullinger um die Ausgabe der Briefe des Ignatius, die Gesner schon in drei Editionen veröffentlicht hatte. Ignatius von Antiochien, gest. um 110 n. Chr., mit den Beinamen Theophoros, Theophilos, ein Eiferer gleich Paulus aus dem Urchristentum, starb als Martyrer unter Trajan in Rom. - C. Geßner übersetzte diese umstrittenen Briefe, Zeugnisse urchristlichen Glaubens. Sie sind von inniger Frömmigkeit, umweht von echtem Christglauben. Epistola John Hoopers, des Bischofs von Gloucester und Worcester, der mit seiner Frau zwei Jahre im Exil in Zürich [1547-1549] verweilte) ad H. Bullinger 
Heilsgedanken Christi, doch außerhalb jeder Dogmatik. Alle großen Geister beschäftigte von jeher die Erkenntnis um die Ontologie, von Aristoteles bis Augustinus, nicht allein Philosophen, Theologen und Ärzte, sondern auch Juristen wie G.BUDÉ ${ }^{49}$. Geßner hatte ein reiches religiöses Innenleben, in welchem er mehr nach dem Sinn der griechischen Väter neigte, wie er dies in seinen Briefen an die Brüder Laelius Socini ${ }^{50}$ und an J.DeleChamps ${ }^{51}$ darlegte. Machtvoll von der Seite der Stoa eines Epiктeт beschäftigten ihn die Aufzeichnungen des Marcus Aurelius Antonius «in semet ipsum ». Es wäre aufschlußreich, Conrad Geßners theologische Werke im Rahmen der Zürcher Geistesgeschichte darzustellen ${ }^{52}$.

Sein geistiges Bewegtsein führte ihn hinaus zum Sehen, Verstehen alles Belebten in der Natur, im Pflanzen-, Tier- und Erdreich, zu einem unablässigen Suchen nach der Wahrheit. Dies war mit ein Ausdruck seiner Frömmigkeit. Wie er immer Empfangender, Mehrer seines Wissens war, ebenso war er bedacht, Gebender zu sein, rückhaltlos allen jenen, die ihn darum baten. Mit Ungeduld erwartete er Briefe, wie er auch eine unübersehbare Menge Briefe schrieb. Sein Freundeskreis und der seiner Adressaten umfaßte schätzungsweise an die 500 .

Überschaut man sachlich die vielen Werke (an die 85 Opera in der Epistola ad Guilielmum Turnerum ${ }^{52}$, Tiguri 1562), erkennt jeder die wohlge-

ex carcere vom 3. September 1553, in Archiv. Eccl. Turicensi, Band Anglicanae litterae (Hanhart, a.a. O., S. 164). C.Gesner widmete dem Martyrer John Hooper, 1555 auf dem Scheiterhaufen, eine ergreifende Elegie, in Neujahrsblatt, herausgegeben von der Stadtbibliothek in Zürich auf das Jahr 1893, von Th. Vetter. Th. Vetter, Joh. Hooper, Bischof von Gloucester und Worcester und seine Beziehungen zu Bullinger und Zürich, in Turicensia, Beiträge zur zürcherischen Geschichte, Zürich 1891, S. 129-144. - P. Tн. Camelot, Ignace d'Antioche Lettres, Texte Grec, Introduction, Traduction et Notes, Paris 1958, $8^{\circ}$. - Vermutlich übertrug Conrad Clauser von Zürich die Briefe des Theophili sexti Antiocheni episcopi de Deo et fide christianorum contra gentes - in der C.Geßner-Ausgabe von 1546. Vollständige Übersicht der Werke C.CuAusers in C. Gessner, Bibliotheca instituta et collecta, Tiguri 1583, S. 161.

49 G. BudÉ, De tranquillitate animi, Parisii 1505.

50 C. Gesneri Epistolae ad Laelii Socini fratres, in Epistolarum medicinalium, Liber quartus, s. HaNhart, a.a. O., S. 349-355.

51 C. Gesneri Epistola: De Constantia in Fide, adversus Apostasiam, in Museum Helveticum, Particula I, Tiguri 1746.

52 H.Buess, in Gesnerus 5 (1948) 1-29.

${ }^{52 a}$ William Turner, 1520-1568, studierte in Cambridge, Dr. Medicinae in Ferrara, dozierte in Oxford, 1557-1559 in Deutschland und Italien, einige Zeit in Basel. Mit C. Geßner stand er seit 1541 in Verbindung, er besuchte ihn in Zürich. Er ist Verfasser des 
ordnete methodische Arbeitsweise, die klare übersichtliche Darstellung. Er besaß in hohem Grade den Sinn für das Wort, den Träger kernhafter Gedanken, den er oft mit griechischem Ausdruck erhellte, selbst ganze Zitate, die ihn als gewandten Humanisten der «bonae litterae» charakterisieren. Er schrieb eine kleine, je nach Federkiel versteifte Humanistenschrift mit oft eigenwilligen Abbreviaturen.

Man nannte Geßner gelegentlich, ohne seine Werke näher zu kennen, einen Kompilator oder Nachschreiber. Dabei war er reich an Einfällen, ein ausgezeichneter Beobachter, begnadet mit einer Kraft, einer Arbeitsspannweite von heute schwer erfaßbarem Umfang im ständigen Nebeneinander heranwachsender Werke. Mit welcher Liebe saß er über den Pergamenten im Ringen um den authentischen Wortlaut, um die Wahrheit der Übersetzung und der Wortbedeutungen. Gewiß darf man ihn nur messen am Maß seiner Zeit; nicht weniges führte darüber hinaus. Das große Wort lag ihm nicht, seine Größe ruhte in ihm selbst, andere sollten sie nicht fühlen. Bei der Ausgeglichenheit des Charakters war er ein Mann des Maßhaltens, «nequid nimis», schweigsam. Den Dingen der Natur stand er demütig gegenüber, dafür um so freier. Von Conrad Geßner muß etwas Strahlendes ausgegangen sein, eine große Güte, Milde, Bescheidenheit, verbunden mit einer offenen Hilfsbereitschaft gegen seine Freunde und Patienten.

Ganz in seine Arbeiten versenkt, lebte er einsam in einem Gelehrtengehäuse, stets auf sich selbst angewiesen, wurde er Jahr um Jahr verschlossener, wortkarg. Die meisten seiner Werke entstanden in den stillen Nachtstunden bei flackerndem Öllämpchen bis ins Frühlicht des anbrechenden Tages. Im Argumentum zum Thesaurus Evonymi Philiatri (Tiguri 1552) sagte er von diesen Nachtstunden: «Haec lucubratio nostra si approbata fuerit viris bonis et eruditis.» Von dieser drückenden Nachtarbeit berichtete er noch 1561 an JoHN CaJus ${ }^{53}$, dazu kam das Ringen um Zeittermine

ersten englischen Kräuterbuches, A New Herball, London, Tome I. 1551, Tome II. 1552, Tome III. 1568, in fol. Ferner: The Rare Treasure of England Bathes, Köln 1587. Mit C.Geßner tauschte er Pflanzen, Tiere, Kuriosa, Fossilien.

${ }^{53}$ Epistola ad Joannem Cajum, Britannus, in Epistolarum medicinalium, Liber tertius, S. 133-134. - John CaJus, Key oder KaYe, 1510-1573, studierte Medizin in Cambridge, in Padua gemeinsam mit A.VeSAL, doktorierte in Bologna, 1547 Mitglied des College of Physicians in London. Er befaßte sich mit den Werken C. Galens, was Geßner besonders interessierte wegen seinen eigenen großen Galen-Ausgaben 1561/62. J. Cajus beschrieb in einem umfangreichen Brief an Geßner alle in England vorkommenden Hunderassen und Spielarten, der, mehrmals gedruckt, als Liber de canibus Britannicis, London 1570, erschien. 
der Buchdrucker, bei engen Wohnverhältnissen, gekürzter Ruhe, ohne Sonne, bei sparsamer Ernährung, all dies mußte einen Abbau am Körper zur Folge haben, und das während fünfundzwanzig Jahren.

In der Bibliotheca universalis (Tiguri 1545) beurteilte er selbst seine Schriften mit leisem Humor: Sie seien unvollkommener, als sie hätten sein sollen, «wenn ich sie zu Hause mit mehr Muße hätte überarbeiten und wie der Bär seine Jungen lecken oder einige Jahre lang beiseite legen können ». Er ging neue Wege, die nicht ausgetreten waren, die er allein zuerst ging in der Planung zum Pflanzenwerk, wobei er im Verlaufe von Jahren eine Reihe von Vorstudien machte. Die zusammengetragenen Materialien wuchsen ins Unübersehbare (schon 1553 besaß er über hundert Abbildungen seltener Pflanzen). Im Jahre 1557 berichtete Geßner an den Lieblingsschüler Caspar WolF ${ }^{54}$ nach Montpellier, er beginne jetzt ein großes Pflanzenwerk. Als eine Zugabe zur Descriptio montis fracti fügte er De raris et admirandis herbis noctu lucentibus, quae lunariae vocantur (Tiguri 1555) bei. Im Winter 1559/60 arbeitete er an den Horti Germaniae (Argentorati 1561); in beinahe allen Briefen von 1559 bis 1565 lesen wir von seinem Wunsch, sein Herbarium und seine Abbildungen zu mehren, die Historia plantarum zu vollenden.

Um 1552 erkrankte Geßner sehr schwer an heftigem Husten, einem Katarrh, wie er an A.P. GASSER ${ }^{55}$ am 19. April 1565 berichtete: "egomet ante annos 9 fere eadem ex contagione laboravi.» Er fühlte sich körperlich geschwächt. Seine auswärtigen Besucher fanden ihn damals blaß und frühzeitig gealtert.

Wegen seiner Armut gelangte er 1553 an Bullinger mit der Bitte um eine Chorherrenpfründe ${ }^{56}$. Wenige Jahre später richtete Geßner an den Bürgermeister und Rat von Zürich im September 1558 ein Gesuch um bessere Besoldung als Stadtarzt: «Nun aber will mir die Sach zu schwär werden, und meine Ämpter darnäben wie sich gebürt versähen, nitt wol mee müg-

54 Epistola ad Casparum Wolphium, in Epistolarum medicinalium, Liber tertius, S. 122-122 b. - J. J. Rousseau fand im Januar 1772 in der Bibliothek des Monsieur DE Lamoignon de Malesherbes en son château de Malesherbes par Etamps, wie er im Briefe vom 19. Januar 1772 an Monsieur de Malesherbes schrieb, von Conrad Gessner, De raris et admirandis herbis noctu lucentibus, quae lunaria vocantur, Tiguri 1555, die Rousseau zu weiteren botanischen Studien begeisterten. - In Lettres sur la botanique par Jean-Jacques Rousseau. Rousseau et la botanique par Bernard Gagnebin, Club des Libraires de France, 1962, S. 181.

55 Epistolarum medicinalium, Liber primus, S. 40 a: ad A.P. Gasserum.

56 Neujahrsblatt der Chorherren (Zürich) auf das Jahr 1782, S. 7. 
lich, dartzu bin ich nit starker Natur, und nümen jung, eines so blöden gesichts, daß ich sämmliche langwirige Arbeiten, die nit allein den Tag, sunder auch ein großen Teil der Nacht erforderen, nitt wol mee erträgen mag.»

In jungen Jahren bestand bei Geßner eine Kurzsichtigkeit, die ihn veranlaßte, sich der «Conspicilli» zu bedienen ${ }^{57}$; aus dem Kurzsehen ergibt sich sein Einfühlungsvermögen für kleinste Details, Blütenstände, Insekten, Fossilien, Mineralien, Kristalle. Er zeichnete ausgezeichnet: herrliche Blattformen, Blüten mit beachtlicher künstlerischer Begabung. Im Briefe an Јон. Kentmann ${ }^{58}$ vom 24.Juni 1564 findet sich dies bestätigt: «sum et ipse subinde in variis plantis depingendis occupatus.»

An Jean BaUHIN ${ }^{59}$ bemerkt er: «Mein Alter fängt mir an beschwerlich zu werden.»

Arg quälte ihn der Ischias, mehr denn drei Wochen, bemerkte er in zwei Briefen an Јон. Сrato von Krafftheim ${ }^{60}$, der ihn zwang, im August 1560 in den Bädern zu Baden Linderung zu suchen.

Die letzten fünf Lebensjahre (1560-1565) gehörten seiner fruchtbarsten Zeit an, in der er aus der Fülle der Aufzeichnungen neue Werke vorbereitete: die Ausgabe des Cl. Galen (1562), den Dioscorides, De rerum fossilium (1565), eine Pharmakologie, ein Insektenbuch, die er beide nicht mehr auszuarbeiten vermochte.

Die Horti Germaniae (1561) enttäuschten ihn schmerzlich wegen des schlechten Druckes, die er mit den Opera des Valerir Cond gleichzeitig in einem stattlichen Folioband herausgab, wußte er doch recht gut, was sich typographisch gehörte.

Nach diesem nicht geringen Ärger sehnte er sich nach seinen Bergen, des «Stubenhockens» müde. Anfangs Juni wanderte Geßner frohgemut mit lieben Freunden ins Engadin, nach Scuol, durchs Val Scarl, überstieg den

57 Hanhart, a. a. O., S. 126: Conspicilli, S. 142: halbblind.

58 Epistolarum medicinalium, Liber quartus: Epistola XVIII. C. Gesnerus ad Joa. Kentmannum, 24.Juni 1564, in Hanhart, a. a. O., S. 333. - Joh. Kentmann, 1518-1565, Arzt in Torgau und Meißen. Als gewiegter Botaniker und Mineraloge schätzte ihn Geßner freundschaftlich verbunden wegen seiner häufigen Tauschsendungen.

${ }^{59}$ Epistolae a Casparo Bauhino, nunc primum editae, Basileae 1598, S. 158: Epistola ad J. Bauhinum. - Jean Bauhin, 1541-1612, studierte in Tübingen, Montpellier und Padua, promovierte 1562, der Ort ist ungewiß, ebenso nicht sicher wird er als Reisebegleiter C. Geßners vermerkt auf dessen Wanderung durch Graubünden nach dem Veltlin im Juni 1561. 1563-1565 weilte er in Lyon, 1568 dozierte er Botanik neben dem Amt als Stadtarzt in Genf, seit 1571 praktizierte er in Montbéliard.

${ }^{60}$ Hanhart, a.a. O., S. 187, 189-192. 
Umbrail hinunter zu den Bagni di Bormeo, die ihm für seine Leiden heilsam schienen. Herrliche Wandertage verlebte er zur Zeit der Vollblüte der Bergflora auf den Hochpässen der Alpen, wo er eifrig nach seltenen Pflanzen suchte ${ }^{61}$. Und auf der Heimreise überraschte ihn die herzenswarme Gastfreundschaft des Arztes Franciscus Portus ${ }^{62}$ in Chiavenna, eines besten Kenners der dortigen Flora.

Der Poesie Geßners wurde kaum je Beachtung geschenkt. Er nannte das Verseschreiben im Brief an Adolph Occo ${ }^{63}$ "Spielwerk der Jugend». Als Dichter in glücktragenden Stunden sah er Visionen - vielleicht blasse, ohne Größe, die zu Idealen schwebten, wertlos, für ihn bedeutend, Gedankenlichen Schaffen suchte er noch Zeit zu beglückender Poesie, jugendlichen Sinnes, ein Schwingen ins Erhabene ${ }^{64}$.

Was in ihm Verse Homers, die Idyllen Theokrits ${ }^{65}$ anregten, brach in seltenen Lichtstunden aus innerem Leuchten zutage.

Es handelt sich zumeist um lateinische Hexameter und griechische Jamben, festliche Gaben zu einem seiner Werke oder als Elegien für Freunde: Huldreich Zwingli, John Hooper, David Kyber ${ }^{66}$, von sinniger Herzlichkeit.

61 Epistolarum medicinalium, Liber tertius, S. 85: C. Gesnerus Cosmae Holtzachio, 18. Juli 1561: "Abfui toto mense in Rhaeticis alpibus vallibusque ad summam usque Tellinam vallem progressus, stirpes et fontes maxime inspiciundi causa, meque exercendi: duobus in locis balneo usus sum ad paucos dies, in uno etiam stillicidio.»Cosmas Holtzach, $† 1595$, studierte in Basel, Dr. Medicinae, Stadtarzt in Schaffhausen, an ihn sind 15 Briefe C. Geßners im Epistolarum medicinalium, Liber tertius, Tiguri 1577 , S. $81 \mathrm{a}-88$.

62 Franciscus Portus, 1511 in Candia auf Kreta, 1581 in Genf. 1561 weilte er als Arzt in Chiavenna, wo er C. Geßner aus seinem Garten das «Apium palustre» verehrte samt Samen. In Horti Geraminae, Argentorati 1561, in Fol. S. 247: «nuper Chiavennae in horto suo Franciscus Portus Cretensi, Graecorum omnium in hodie facile doctis simus ostendit et semina communicavit.»- Ebenso in D.Fretz, Conrad Geßner als Gärtner, Zürich 1948, S. 176. - Epistolarum medicinalium, Liber tertius, S. 133-133 a. C. Gesnerus Francisco Porto Cretensi, Graecarum literarum interpreti consummatissimo vom 6. Febr. 1563. - Epistolarum medicinalium, Liber primus, S. 24 a, Epistola: C. Gesnerus ad A.P. Gasserum, 27.Februar 1563, betreffend F.Portus: «D.Portus amicus meus, Cretensi, qui Genevae nunc habitat. »

63 Hanhart, a.a. O., S. 275-279: Brief C. Geßners an Adolph Occo vom 4. Dezember 1565.

64 Epistolarum medicinalium: Nach der Dedicatoria, von Caspar Wolf gesammelte Poesie.

65 C. Gesneri Translatio in latine Theocriti Bionis Moschi bucolicorum graecorum idylliae, Zentralbibliothek Zürich, in C 86 a (Variaband).

66 In Epistola C.Gesneri ad G. Turnerum (Tiguri 1562) Nr. 4.1: in auroris mortem Idyllium lugubre Graecum condidi (für DAvid KYBER). 
Alle diese Verse finden sich eingestreut gleich Blüten auf den ersten Blättern seiner Werke, übersehen, vergessen im Papier.

Wie herzlich lachte Geßner, da sein Freund Jаков RuefF ${ }^{67}$, der Chirurgus Turicensis, zu Zürich 1550 in seinem Spiel Adam und Heva im Paradies gleich mit der ganzen Geßnerischen Tierwelt «in Figuris» aufzog.

Im Sommer 1559 übergab Geßner dem Kaiser Ferdinand I. in Augsburg persönlich das Fischbuch. Bei diesem Anlaß mag der Kaiser zu seinem Leibarzt Joh. Сrato von Krafftheim jenes Lob ausgesprochen haben: «Credas, Gesnerus tota probitas!» ${ }^{68}$

Der Tod seiner achtzigjährigen Mutter im April 1564 erschütterte ihn, den Ruhm ihres lieben Sohnes, den Wappenbrief der Geßner vom 3. April 1564, dürfte sie kaum erlebt haben.

Das lebensnahe Bildnis des edlen Conrad Geßner schuf Toвias SтімMER $^{69}$, ein Meisterwerk von erhabenem Eindruck. Das Brustbild kommt einer Urkunde gleich, einem Schicksalsspiegel in seiner Geistigkeit. Sein Antlitz ist schmal, kein Zug erscheint zufällig, müde, gütige Augen blicken dich an. Die linke Stirnader tritt leicht hervor unter dem Barett, die Wangen sind eingefallen, der Mund erscheint schmal, man wäre versucht zu sagen, er sei zahnlos, zusammengepreßt. Der Schnurrbart und der wallende Bart schimmern ergraut.

Die linke Hand umfaßt den rechten Zeigefinger, den Schreibefinger, warum? (Arthritis ?)

Die erhabene Gestalt gekleidet in die spätgotische Schaube. Es ist das Bild eines gelehrten, asketischen Mannes, voll Güte des inneren Friedens, fromm in sich ergeben.

Die Haltung vermittelt in ihren Konturen einen deutlichen psychologischen Einblick in das Wesen dieses Mannes.

Das Bild entstand vermutlich im Frühjahr 1562, zur Zeit, da Geßner im Auftrage von Alexander Peyer, des Bürgermeisters und Rats von Schaffhausen, die Apotheken visitierte.

67 Jаков Baechtold, Geschichte der deutschen Literatur in der Schweiz, Frauenfeld 1892, S. 329-330 (J. Rueff).

68 HanhaRT, a. a. O., S. 251-263.

69 Tobias Stimmer, 1539-1584, Historisch-Biographisches Lexikon der Schweiz, Band 6, S. 553-554. 
Das Wappen Geßners und die Schrift dürften erst 1564 hinzugemalt sein:

Conradus Gesnerus Tigurinus Medicus

et Philosophiae Interpres. Anno Aetatis suae xlviil.

Anno Salutis molxiili Nonis Martiis.

Das Porträt Geßners, von einer unerhörten Lebenswirklichkeit, erlaubt beinahe eine klinische Diagnose: Er ist früh gealtert in rastloser Arbeit, abgezehrt durch jahrelange Entbehrungen, verbraucht in der Pflicht seines Amtes, aber noch voll Selbstvertrauen, in sich gekehrt, zwischen Staunen und Lächeln, blickt er in die Ferne. Dieser selbstlose Mann des ehrenfesten Zürich schenkte der gelehrten Welt unter großen Opfern in drei Jahrzehnten hervorragende Beiträge von bleibendem Wert aus manchen Wissenszweigen. Ein Rest verblaßten Ruhmes verblieb ihm in der heutigen wissenschaftlichen Botanik in den Gesnerianae.

Conrad Geßner ist einer der ganz großen Meister unseres Zürich, dessen Andenken wir bedenken und bewahren müssen. 